\title{
Propiedades acústicas de madera de Fraxinus americana y Fraxinus uhdei
}

\author{
Javier Ramón Sotomayor Castellanos ${ }^{1 \star}$, José María Villaseñor Aguilar ${ }^{1}$ \\ ${ }^{1}$ Universidad Michoacana de San Nicolás de Hidalgo, Morelia, México.
}

\begin{abstract}
RESUMEN El objetivo de la investigación fue determinar la velocidad del ultrasonido, el módulo dinámico, la radiación y la impedancia acústicas, de la madera de Fraxinus americana y Fraxinus uhdei. La metodología consistió en los siguientes pasos: se prepararon para cada especie, treinta y dos probetas con dimensiones de $2 \mathrm{~cm}$ x $2 \mathrm{~cm}$ x $32 \mathrm{~cm}$. Se calcularon la densidad aparente y el contenido de humedad de la madera y se realizaron pruebas de ultrasonido. Se midió la velocidad del ultrasonido y se determinaron el módulo dinámico, la impedancia acústica y el coeficiente de radiación acústica. Las maderas de Fraxinus americana y Fraxinus uhdei tienen densidad aparente similar. El coeficiente de radiación acústica es similar para ambas especies. Sin embargo, su módulo dinámico y sus índices de rendimiento acústico son diferentes. Las características acústicas de la madera de Fraxinus americana y Fraxinus uhdei sitúan favorablemente a estas especies para que usos tradicionales se diversifiquen al incorporarlas como material de ingeniería en la industria de transformación.
\end{abstract}

Palavras-chave: densidad de la madera, velocidad del ultrasonido, módulo dinámico, radiación acústica, impedancia acústica.

\section{Acoustic properties of Fraxinus americana and Fraxinus uhdei wood}

\begin{abstract}
The research goal was to determine the ultrasound speed, the dynamic modulus, the acoustic radiation and impedance of Fraxinus americana and Fraxinus uhdei wood. The methodology included the following: For each species, thirtytwo specimens were prepared with dimensions of $2 \mathrm{~cm} \times 2 \mathrm{~cm} \times 32 \mathrm{~cm}$. The apparent wood density and moisture content were calculated and ultrasound tests were achieved. The ultrasound speed was measured and the dynamic modulus, the acoustic radiation and the impedance were calculated. The Fraxinus americana and Fraxinus uhdei woods have a similar density. The acoustic radiation is alike between species. However, the dynamic modulus and its acoustic performance indexes are different. The acoustic characteristics of the Fraxinus americana and Fraxinus uhdei woods favorably set these species to diversify their traditional uses including them as engineering material in the transformation industry.
\end{abstract}

Keywords: wood density, ultrasound speed, dynamic modulus, acoustic radiation, acoustic impedance.

\section{Introducción}

En la literatura científica, la caracterización del comportamiento acústico de maderas empleadas tradicionalmente o con potencial para la elaboración de instrumentos musicales, puede ser consultada entre otros, en Ilic (2003); Bucur (2006); Spycher et al. (2008); Sedik et al. (2010); Brémaud et al. (2012) y Fouilhé et al. (2012). Estos autores determinan las principales características y los índices de calidad acústicos de maderas a nivel internacional. En el mismo contexto, Obataya et al.
(2000); Brancheriau et al. (2006); Wegst (2006); Traoré et al. (2010); Roohnia et al. (2011) y Smardzewski et al. (2014) investigan la estructura anatómica y las propiedades de anisotropía de la madera y su relación respecto a sus propiedades acústicas. Estos trabajos identifican igualmente especies de maderas con usos actuales y potenciales para su incorporación como elementos estructurales y estéticos en instrumentos musicales.

Este enfoque de clasificación de una especie de madera para su recomendación en la elaboración de instrumentos *Corresponding author: madera999@yahoo.com 
musicales, es de carácter práctico y encuentra sustento en que, para la selección de una madera, el fabricante se guía preferentemente por indicadores visuales, que son expresión de la estructura macro anatómica de una especie en particular (BUKSNOWITZA; TEISCHINGER, 2007).

Para una correcta evaluación de la madera en aplicaciones acústicas, son necesarios su densidad aparente y su módulo dinámico (SPYCHER et al., 2008) y la velocidad del ultrasonido (BRANCHERIAU et al., 2010). Además, Roohnia et al. (2011) y Brémaud et al. (2012) proponen como indicadores de la calidad acústica de la madera, el coeficiente de radiación acústica y la impedancia acústica. Estos parámetros explican las características de resonancia de una madera y son necesarios en el diseño no solo de instrumentos musicales, sino también en componentes donde las cualidades de aislamiento y/o resonancia acústica son deseables, por ejemplo, en el cálculo de elementos estructurales y/o arquitectónicos en edificaciones con madera.

La densidad aparente de la madera es el parámetro que se considera usualmente como buen predictor de las características mecánicas de la madera. Este indicador es una característica física y es función de la estructura anatómica del material, específicamente de su porosidad y del acomodo de los tejidos de crecimiento (BUCUR, 2006). La densidad aparente de la madera es una magnitud intensiva, que está relacionada con la porosidad de una madera en particular y con su contenido de humedad al momento de la medición (FUENTES, 2000).

La velocidad del ultrasonido y el módulo dinámico de la madera dependen de la temperatura y del contenido de humedad de la madera. Igualmente, estos parámetros dependen del tiempo y de las condiciones de ensayo en las cuales se determinaron (SHMULSKY; JONES, 2011). La velocidad del ultrasonido viajando a través de la madera explica la capacidad del material para trasmitir una onda mecánica, en este caso una onda acústica (ROOHNIA et al., 2011). El módulo dinámico es de igual forma una magnitud intensiva de la madera y representa la cantidad de energía elástica que puede almacenar un volumen de madera (SHMULSKY; JONES, 2011). Ambos parámetros dependen, entre otros factores, del contenido de humedad, de la anisotropía y del método de determinación.

El coeficiente de radiación acústica es un descriptor de la señal acústica y del mecanismo de disipación de energía sonora, es decir, del sonido (BRÉMAUD, 2012). Por su parte la impedancia acústica relaciona la facilidad de la transmisión de una vibración de un medio a otro (SPYCHER et al., 2008). Ambos parámetros son considerados como índices de rendimiento acústico de la madera.

Existen pocos precedentes sobre la caracterización mecánica de las especies Fraxinus americana y Fraxinus uhdei. Sus características elásticas y acústicas están reportadas por Sotomayor (2015). Resultados experimentales de las propiedades acústicas y sobre la calidad de maderas mexicanas con uso y potencial para elaborar instrumentos musicales son reportados por Sotomayor; Bocanegra (2009) y Sotomayor et al. (2010). Respecto a Fraxinus americana, el Manual de la madera del Laboratorio de Productos Forestales de Los Estados Unidos de América (FOREST PRODUCTS LABORATORY, 2010) reporta sus características tecnológicas. Para la madera de Fraxinus uhdei, no se encontró información al respecto.

Las maderas de Fraxinus americana y Fraxinus uhdei se emplean y se comercializan distinguiéndolas como fresno mexicano (Fraxinus uhdei) o fresno americano (Fraxinus americana) (TAMARIT; LÓPEZ, 2007; SILVA et al., 2010). Ambas especies se utilizan tradicionalmente en la manufactura de artesanías y juguetes, instrumentos musicales, implementos agrícolas y mangos para herramientas, muebles finos y artículos deportivos. 
El objetivo de la investigación fue determinar la velocidad del ultrasonido, el módulo dinámico, la radiación y la impedancia acústicas, de la madera de Fraxinus americana y Fraxinus uhdei.

\section{Material y Métodos}

El material experimental consistió en madera de Fraxinus americana L. y Fraxinus uhdei (Wenz.) Lingelsh., recolectada en terrenos forestales del Estado de Michoacán $\left(19^{\circ} 10^{\prime} 07^{\prime \prime} \mathrm{N}, 101^{\circ} 53^{\prime} 59^{\prime \prime} \mathrm{O}\right)$, México. Las especies fueron identificadas en el Laboratorio de Mecánica de la Madera, de la Facultad de Ingeniería en Tecnología de la Madera, de la Universidad Michoacana de San Nicolás de Hidalgo. A partir de 16 piezas de madera aserrada, se prepararon para cada especie, 32 probetas con dimensiones de $2 \mathrm{~cm} \times 2 \mathrm{~cm} \mathrm{x}$ $32 \mathrm{~cm}$, orientadas respectivamente en las direcciones radial, tangencial y longitudinal del plano leñoso, de acuerdo a las recomendaciones de la norma ISO 3129:2012 de la Organización Internacional de Normalización - ISO (ISO, 2012). La madera se estabilizó durante 6 meses en una cámara de acondicionamiento con una temperatura de $20^{\circ} \mathrm{C}\left( \pm 1^{\circ} \mathrm{C}\right)$ y una humedad relativa de $60 \%( \pm 2 \%)$, hasta que alcanzó un peso constante.

Las pruebas de ultrasonido consistieron en suministrar un impulso ultrasónico en transmisión directa en la dirección longitudinal de la probeta. La velocidad del ultrasonido se calculó con el cociente entre la longitud de las probetas y el tiempo de transmisión medido con el aparato Sylvatest ${ }^{\circledR}$ posicionado en un dispositivo para pruebas no destructivas diseñado ad-hoc (Figura 1). De aquí en adelante, a los parámetros determinados, se les asocia el subíndice "us" para identificarlos como derivados de pruebas de ultrasonido.

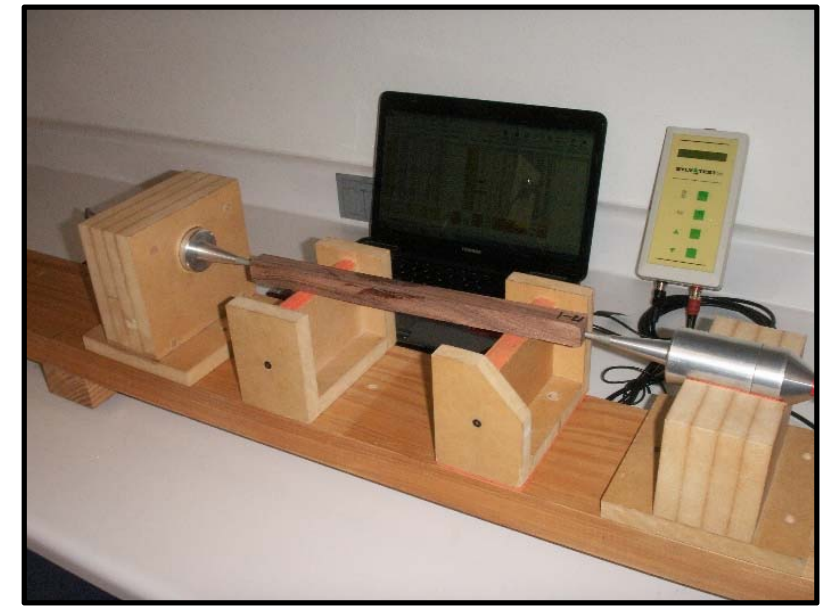

Figura 1. Dispositivo experimental para las pruebas de ultrasonido.

Figure 1. Experimental device for the ultrasound tests.

El contenido de humedad se determinó por el método de diferencia de pesos con grupos complementarios de probetas con dimensiones de $2 \mathrm{~cm} \times 2 \mathrm{~cm} \times 6 \mathrm{~cm}$ en las direcciones radial, tangencial y longitudinal respectivamente de acuerdo con la norma ISO 13061-1:2014 (ISO, 2014b). El contenido de humedad de la madera se calculó con la Equación 1 (ISO, 2014b).

$$
\mathrm{CH}=\left[\left(\mathrm{P}_{\mathrm{CH}}-\mathrm{P}_{\mathrm{A}}\right) / \mathrm{P}_{\mathrm{A}}\right] \times 100 \quad \text { Equación } 1
$$

En que: $\mathrm{CH}=$ Contenido de humedad, \%; $\mathrm{P}_{\mathrm{CH}}=$ Peso de la probeta a un contenido de humedad $\mathrm{CH}, \mathrm{kg} ; \mathrm{P}_{\mathrm{A}}=$ Peso de la probeta en estado anhidro, $\mathrm{kg}$.

La densidad aparente de la madera a un contenido de humedad CH se calculó con la Equación 2 de acuerdo con la norma ISO 13061-2:2014 (ISO, 2014a).

$$
\rho_{\mathrm{CH}}=\mathrm{P}_{\mathrm{CH}} / \mathrm{V}_{\mathrm{CH}} \quad \text { Equación } 2
$$

En que: $\rho_{\mathrm{CH}}=$ Densidad aparente, $\mathrm{kg} / \mathrm{m}^{3} ; \mathrm{P}_{\mathrm{CH}}=$ Peso de la probeta a un contenido de humedad $\mathrm{CH}, \mathrm{kg} ; \mathrm{V}_{\mathrm{CH}}=$ Volumen de la probeta a un contenido de humedad $\mathrm{CH}, \mathrm{m}^{3}$. 
El módulo dinámico se calculó con la Equación 3 propuesta por Brémaud (2012).

$$
\mathrm{E}_{\mathrm{us}}=\mathrm{v}_{\mathrm{us}}^{2} \rho_{\mathrm{CH}} \quad \text { Equación } 3
$$

En que: $\mathrm{E}_{\mathrm{us}}=$ Módulo dinámico, $\mathrm{N} / \mathrm{m}^{2} ; \mathrm{v}_{\mathrm{us}}=$ Velocidad del ultrasonido, $\mathrm{m} / \mathrm{s} ; \rho_{\mathrm{CH}}=$ Densidad aparente, $\mathrm{kg} / \mathrm{m}^{3}$.

El coeficiente de radiación acústica se calculó de acuerdo con la Equación 4 propuesta por Brémaud (2012).

$$
\mathrm{R}_{\mathrm{us}}=\mathrm{v}_{\mathrm{us}} / \rho_{\mathrm{CH}} \quad \text { Equación } 4
$$

En que: $\mathrm{R}_{\mathrm{us}}=$ Coeficiente de radiación acústica, $\mathrm{m}^{4} / \mathrm{kg} \mathrm{s} ; \mathrm{v}_{\mathrm{us}}=$ Velocidad del ultrasonido, $\mathrm{m} / \mathrm{s} ; \rho_{\mathrm{CH}}=$ Densidad aparente, $\mathrm{kg} / \mathrm{m}^{3}$.

La impedancia acústica se calculó con la Equación 5 propuesta por Brémaud (2012).

$$
\mathrm{z}_{\mathrm{us}}=\mathrm{v}_{\mathrm{us}} \rho_{\mathrm{CH}} \quad \text { Equación } 5
$$

En que: $\mathrm{z}_{\mathrm{vt}}=$ Impedancia acústica, $\mathrm{kg} / \mathrm{m}^{2} \mathrm{~s} ; \mathrm{v}_{\mathrm{us}}=$ Velocidad del ultrasonido, $\mathrm{m} / \mathrm{s} ; \rho_{\mathrm{CH}}=$ Densidad aparente, $\mathrm{kg} / \mathrm{m}^{3}$.

Se determinaron los estadísticos media aritmética y coeficiente de variación. Una vez que se verificó que las muestras provenían de distribuciones normales, se diseñó un experimento siguiendo las recomendaciones de Gutiérrez; de la Vara (2012). Las variables de respuesta fueron la densidad aparente y la velocidad del ultrasonido. El módulo dinámico, el coeficiente de radiación acústica y la impedancia acústica se analizaron como variables derivadas. La especie se consideró el factor de variación.

El experimento consistió en pruebas $t$ de Student para muestras independientes de diferencias de medias para un nivel de confianza de $95 \%$. Se efectuaron pruebas con 32 réplicas (probetas por especie), totalizando 64 muestras obser- vadas para cada una de las variables de respuesta. Los cálculos estadísticos fueron realizados con el programa Statgraphics.

Para ilustrar el posicionamiento de las características acústicas de la madera de Fraxinus americana y Fraxinus uhdei, en relación a las de otras especies reportadas por Sotomayor (2015), se determinaron regresiones lineales con los datos bibliográficos y se compararon con los resultados de esta investigación.

\section{Resultados y Discusión}

Las densidades aparentes de Fraxinus americana y Fraxinus uhdei corresponden a contenidos de humedad similares y homogéneos al interior de las muestras (Tabla 1). El contenido de humedad de la madera se mantuvo constante durante la realización de las pruebas de ultrasonido. Considerando la magnitud y el intervalo de variación del contenido de humedad de la madera, su influencia en la velocidad del ultrasonido es mínima (OZYHAR et al., 2013). De tal forma que este parámetro no intervino en la variación de los resultados. En este estado de humedad, la densidad aparente para ambas maderas clasifica como media de acuerdo con Sotomayor; Ramírez (2013).

Los valores promedio de la densidad aparente, la velocidad del ultrasonido y el módulo dinámico para las maderas de Fraxinus americana y Fraxinus uhdei, son congruentes con los parámetros de maderas con densidades similares, reportados en el Banco FITECMA de características físico-mecánicas de maderas mexicanas (SOTOMAYOR, 2015). Igualmente, los valores del coeficiente de radiación acústica y de la Impedancia acústica de Fraxinus americana y Fraxinus uhdei se sitúan en el rango de los reportados por Roohnia et al. (2011). 
Tabla 1. Propiedades acústicas de Fraxinus americana y Fraxinus uhdei.

Table 1. Acoustic properties of Fraxinus americana and Fraxinus uhdei.

\begin{tabular}{ccccccc}
\hline & $\begin{array}{c}\mathrm{CH} \\
(\%)\end{array}$ & $\begin{array}{c}\rho_{\mathrm{CH}} \\
\left(\mathrm{kg} / \mathrm{m}^{3}\right)\end{array}$ & $\begin{array}{c}\mathrm{V}_{\mathrm{us}} \\
(\mathrm{m} / \mathrm{s})\end{array}$ & $\begin{array}{c}\mathrm{E}_{\text {us }} \\
\left(\mathrm{N} / \mathrm{m}^{2}\right)\end{array}$ & $\begin{array}{c}\mathrm{R}_{\text {us }} \\
\left(\mathrm{m}^{4} / \mathrm{kg} \mathrm{s}\right)\end{array}$ & $\begin{array}{c}\mathrm{Z}_{\mathrm{us}} \\
\left(\mathrm{kg} / \mathrm{m}^{2} \mathrm{~s}\right)\end{array}$ \\
\hline \multicolumn{7}{c}{ Fraxinus americana } \\
$\overline{\mathrm{x}}$ & 10.95 & 631 & 4655 & 13697 & 7.40 & 2.94 \\
$\mathrm{CV}$ & 9.22 & 5.41 & 5.63 & 11.63 & 8.39 & 7.19 \\
& \multicolumn{5}{c}{ Fraxinus uhdei } \\
$\overline{\mathrm{x}}$ & 10.70 & 625 & 4453 & 12451 & 7.14 & 2.78 \\
$\mathrm{CV}$ & 11.12 & 4.76 & 5.85 & 14.01 & 6.48 & 8.61 \\
\hline
\end{tabular}

En que: $\mathrm{CH}=$ Contenido de humedad; $\rho_{\mathrm{CH}}=$ Densidad aparente; $\mathrm{v}_{\mathrm{us}}=$ Velocidad del ultrasonido; $\mathrm{E}_{\mathrm{us}}=$ Módulo dinámico $\left(\mathrm{x} 10^{6}\right) ; \mathrm{R}_{\mathrm{us}}=$ Coeficiente de radiación acústica; $\mathrm{z}_{\mathrm{us}}=$ Impedancia acústica $\left(\mathrm{x} \mathrm{10} 0^{3}\right) ; \overline{\mathrm{x}}=$ Media; $\mathrm{CV}=$ Coeficiente de variación en porciento.

Para las dos especies, el coeficiente de variación de $\rho_{\mathrm{CH}}$ es menor que el reportado por Brémaud et al. (2012) para maderas angiospermas $(C V=26 \%)$. El coeficiente de variación de la velocidad del ultrasonido en la madera es de la misma magnitud que la reportada por Sotomayor et al. (2010). En cambio, el coeficiente de variación del módulo dinámico es menor que el propuesto por los autores citados $(\mathrm{CV}=33 \%)$.

La densidad aparente de la madera no presenta una diferencia entre las dos especies $(P=0.453 ; \alpha=0.05)$. Sin embargo, la velocidad del ultrasonido, fue estadísticamente diferente $(P<0.05 ; \alpha=0.05)$ entre la madera de Fraxinus americana y la de Fraxinus uhdei.

La Figura 2 presenta el posicionamiento de la velocidad del ultrasonido de Fraxinus americana y Fraxinus uhdei, en relación a la dispersión de las velocidades de 23 maderas reportadas por Sotomayor (2015), todas en función de la densidad aparente. La numeración corresponde a las 23 especies presentadas en la Tabla 2.

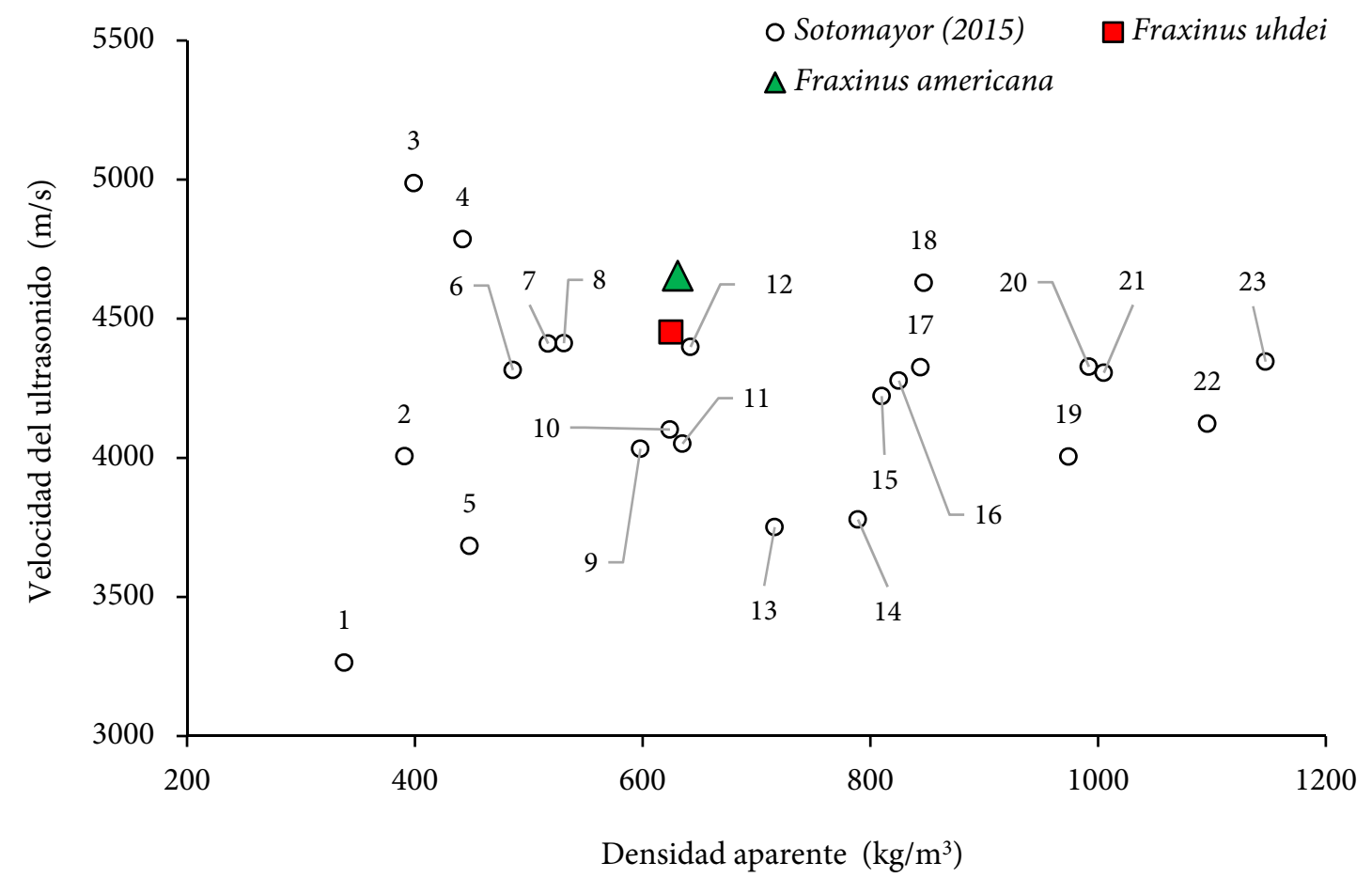

Figura 2. Velocidad del ultrasonido de Fraxinus americana y Fraxinus uhdei en relación a la velocidad de 23 maderas (SOTOMAYOR, 2015).

Figure 2. Ultrasound speed of Fraxinus americana y Fraxinus uhdei related to the speed of 23 woods (SOTOMAYOR, 2015). 
Tabla 2. Especies y numeración utilizada en la Figura 2 (SOTOMAYOR, 2015).

Table 2. Species and numbering used in Figure 2 (SOTOMAYOR, 2015).

\begin{tabular}{|c|c|c|c|}
\hline No. & Especie & No. & Especie \\
\hline 1 & $\begin{array}{l}\text { Spathodea } \\
\text { campalunata }\end{array}$ & 13 & Andira inermis \\
\hline 2 & $\begin{array}{l}\text { Gyrocarpus } \\
\text { americanus }\end{array}$ & 14 & Psidium sartorianum \\
\hline 3 & Abies religiosa & 15 & Juglans pyriformis \\
\hline 4 & Tilia mexicana & 16 & Caesalpinia platyloba \\
\hline 5 & $\begin{array}{l}\text { Enterolobium } \\
\text { cyclocarpum }\end{array}$ & 17 & Albizia plurijuga \\
\hline 6 & Cupressus lindleyi & 18 & Quercus spp \\
\hline 7 & Cedrela odorata & 19 & Lysiloma acapulcensis \\
\hline 8 & $\begin{array}{l}\text { Swietenia ma- } \\
\text { crophylla }\end{array}$ & 20 & Cordia elaeagnoides \\
\hline 9 & $\begin{array}{c}\text { Tabebuia } \\
\text { donnell-smithii }\end{array}$ & 21 & $\begin{array}{l}\text { Acosmium } \\
\text { panamense }\end{array}$ \\
\hline 10 & Dalbergia paloescrito & 22 & Tabebuia chrysantha \\
\hline 11 & Tabebuia rosea & 23 & Dalbergia granadillo \\
\hline 12 & Fagus mexicana & & \\
\hline
\end{tabular}

La velocidad del ultrasonido de Fraxinus americana y Fraxinus uhdei no depende de la densidad aparente de la madera (Figura 2), resultado que coincide con el reportado por Baar et al. (2012). Tomando como referencia la densidad de la madera, para un rango entre 625 y $631 \mathrm{~kg} / \mathrm{m}^{3}$ correspondiente al de Fraxinus americana y Fraxinus uhdei, la velocidad del ultrasonido las posiciona por debajo de las maderas de Abies religiosa (3) y Tilia mexicana (6). Igualmente, la madera de Quercus spp (18), tiene una velocidad similar, pero con una densidad mayor. Con excepción de Abies religiosa, Tilia mexicana y Quercus spp las maderas tropicales (Tabla 2), reportadas por Sotomayor (2015), tienen una velocidad del ultrasonido menor a las especies aquí reportadas.

La Figura 3, presenta el posicionamiento del módulo dinámico de Fraxinus americana y Fraxinus uhdei, en relación a la dispersión de los módulos dinámicos de 23 maderas (SOTOMAYOR, 2015), todos en función de la densidad aparente. El módulo dinámico de Fraxinus americana y Fraxinus uhdei, posiciona a estas especies proporcionalmente cerca de la tendencia de las maderas tropicales, la cual relaciona el módulo dinámico con la densidad aparente. Esta dependencia es explicada por el modelo de regresión con un coeficiente de determinación $\left(\mathrm{R}^{2}\right)$ satisfactorio. Este corolario confirma a la densidad aparente de la madera como un buen predictor de su módulo dinámico (KEUNECKE et al., 2007).

Un criterio de selección para una madera con propiedades de resonancia, es decir, con aptitudes para emplearla en instrumentos musicales es la combinación de una baja densidad con un alto módulo dinámico (BUCUR, 2006; YOSHIKAWA, 2007; YOSHIKAWA; WALTHAM, 2014). En la Figura 3, esta tendencia se indica con la regresión lineal la cual tiende a ir de un módulo dinámico alto, es decir, desde la derecha del eje horizontal, correspondiente a una densidad alta, hacia uno bajo, o hacia la izquierda. Igualmente, en el eje vertical, correspondiente al módulo dinámico, la recta recorre desde abajo, hacia arriba. De tal forma, que las maderas de Fraxinus americana y Fraxinus uhdei se sitúan favorablemente en comparación con las especies reportadas por SOTOMAYOR (2015) y que se reportan con potencial para su empleo en la manufactura de instrumentos musicales por de la Paz et al. (2002).

La Figura 4 presenta el posicionamiento de la impedancia acústica de Fraxinus americana y Fraxinus uhdei, en relación a la dispersión y a la tendencia del coeficiente de radiación acústica de 23 maderas reportadas por Sotomayor (2015).

La correlación permite visualizar el posicionamiento de la impedancia acústica de la madera, en relación al coeficiente de radiación acústica. Su coeficiente de determinación $\left(\mathrm{R}^{2}\right)$ es satisfactorio. Los valores la impedancia acústica de Fraxinus americana y Fraxinus uhdei se sitúan cerca de la tendencia principal, en correspondencia con su coeficiente de radiación. Si bien que estas maderas clasifican como de densidad media, su capacidad para generar energía acústica, percibida como 
sonido irradiado, es cercana a la de maderas Tabebuia donnell-smithii (9), Dalbergia paloescrito (10), Tabebuia rosea
(11) y Fagus mexicana (12), maderas de uso en instrumentos musicales (TAMARIT; LÓPEZ, 2007).

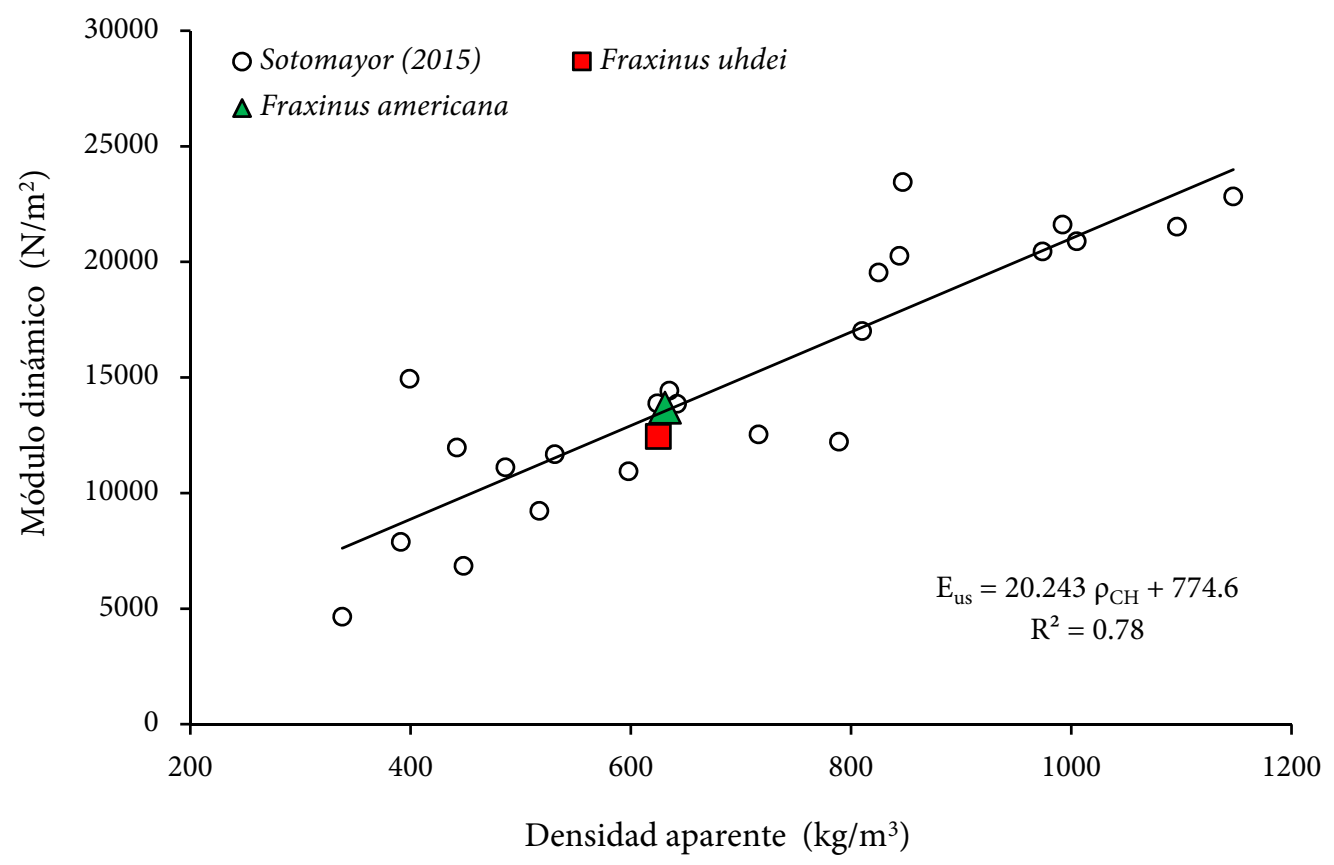

Figura 3. Módulo dinámico de Fraxinus americana y Fraxinus uhdei en relación al módulo dinámico de 23 maderas (SOTOMAYOR, 2015).

Figure 3. Dynamic modulus of Fraxinus americana and Fraxinus uhdei related to the dynamic modulus of 23 woods (SOTOMAYOR, 2015).

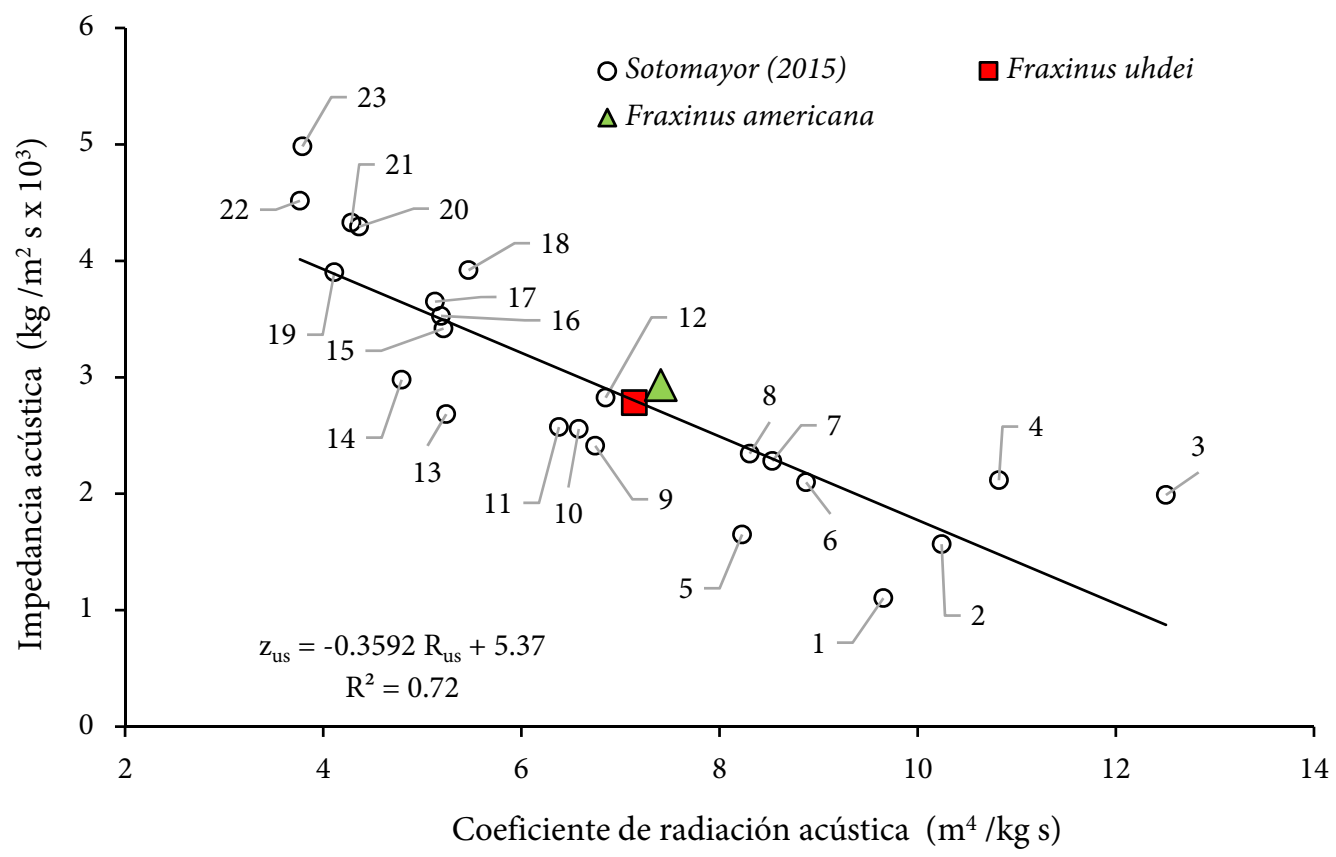

Figura 4. Impedancia acústica de Fraxinus americana y de Fraxinus uhdei en relación al coeficiente de radiación acústica de 23 maderas (SOTOMAYOR, 2015).

Figure 4. Acoustic impedance of Fraxinus Americana and Fraxinus uhdei related to the coefficient of acoustic radiation of 23 woods (SOTOMAYOR, 2015). 


\section{Conclusiones}

Las características acústicas de la madera de Fraxinus americana y Fraxinus uhdei sitúan favorablemente a estas especies para que usos tradicionales se diversifiquen al incorporarlas como material de ingeniería en la industria de transformación. No obstante, la selección de una madera para su empleo aún más específico como un componente estructural de un instrumento musical, necesita de considerar otras propiedades de la madera.

Las maderas de Fraxinus americana y Fraxinus uhdei tienen densidad aparente similar. El coeficiente de radiación es similar para ambas especies. Sin embargo, su módulo dinámico y sus índices de rendimiento acústico son diferentes. El posicionamiento sugerido de Fraxinus americana y Fraxinus uhdei en esta investigación se delimita por su velocidad del ultrasonido y módulo dinámico completado por los índices de rendimiento acústico de radiación y de impedancia acústica.

\section{Agradecimientos}

La investigación estuvo patrocinada por la Coordinación de la Investigación Científica, de la Universidad Michoacana de San Nicolás de Hidalgo, Morelia, México.

\section{Referencias}

BAAR, J.; TIPPNER, J.; GRYC, V. The influence of wood density on longitudinal wave velocity determined by the ultrasound method in comparison to the resonance longitudinal method. European Journal of Wood and Wood Products, v. 70, n. 5, p. 767-769. 2012.

BRANCHERIAU, L.; BAILLÈRES, H.; DÉTIENNE, P.; GRIL, J.; KRONLAND, R. Key signal and wood anatomy parameters related to the acoustic quality of wood for xylophone-type percussion instruments. Journal of Wood Science, v. 52, n. 3, p. 270-273. 2006.
BRANCHERIAU, L.; KOUCHADE, C.; BRÉMAUD, I. Internal friction measurement of tropical species by various acoustic methods. Journal of Wood Science, v. 56, n. 5, p. 371-379. 2010 .

BRÉMAUD, I. Acoustical properties of wood in string instruments soundboards and tuned idiophones: Biological and cultural diversity. Journal of the Acoustical Society of America, v. 131, n. 1, p. 807-818. 2012.

BRÉMAUD, I.; EL KAÏM, Y.; GUIBAL, D.; MINATO, K.; THIBAUT, B.; GRIL, J. Characterization and categorization of the diversity in viscoelastic vibrational properties between 98 wood types. Annals of Forest Science, v. 69, n. 3), p. 373386. 2012.

BUCUR, V. Acoustics of wood. Berlin, Springer-Verlag: 2006.

BUKSNOWITZA, C.; TEISCHINGER, A. Resonance wood [Picea abies (L.) Karst.] evaluation and prediction of violin makers' quality-grading. Journal of the Acoustical Society of America, v. 121, n. 4, p. 2384-2395. 2007.

DE LA PAZ PÉREZ OLVERA, C.; VÉLEZ JIMÉNEZ, S.; SERRANO GARCÍA, A.; ROCHIN GARCÍA, B. La madera de las guitarras de Paracho, Michoacán. ContactoS, v. 44, p. 4553. 2002.

FOREST PRODUCTS LABORATORY. Wood handbook. Wood as an engineering material. Madison, Forest Products Laboratory: 2010.

FOUILHÉ, E.; HOUSSAY, A.; BRÉMAUD, I. Dense and hard woods in musical instrument making: comparison of mechanical properties and perceptual "quality" grading. In: Proceedings of the Acoustics 2012 Nantes Conference. Nantes. p. 2689-2695. 2012.

FUENTES SALINAS, M. Estimación del Punto de Saturación de la Fibra (PSF) de las maderas. Revista Chapingo. Serie Ciencias Forestales y del Ambiente, v. 6, n. 1, p. 79-81. 2000.

GUTIÉRREZ PULIDO, H.; DE LA VARA SALAZAR, R. Análisis y diseño de experimentos. México, Mc Graw Hill: 2012.

ILIC, J. Dynamic MOE of 55 species using small wood beams. Holz als Roh- und Werkstoff, v. 61, n. 3, p. 167-172. 2003. 
ISO 13061-2:2014. Wood. Determination of density for physical and mechanical tests. Geneva: International Organization for Standardization. 2014a. 5 p.

ISO 13061-1:2014. Wood. Determination of moisture content for physical and mechanical tests. Geneva: International Organization for Standardization. 2014b. 4 p.

ISO 3129:2012. Wood. Sampling methods and general requirements for physical and mechanical testing of small clear wood specimens. Geneva: International Organization for Standardization. 2012.9 p.

KEUNECKE, D.; SONDEREGGER, W.; PERETEANU, K.; LÜTHI, T.; NIEMZ, P. Determination of Young's and shear moduli of common yew and Norway spruce by means of ultrasonic waves. Wood Science and Technology, v. 41, n. 4, p. 309-327. 2007.

OBATAYA, E.; ONO T.; NORIMOTO, M. Vibrational properties of wood along the grain. Journal of Materials Science, v. 35, n. 12, p. 2993-3001. 2000.

OZYHAR, T.; HERING, S.; SANABRIA, S. J.; NIEMZ, P. Determining moisture-dependent elastic characteristics of beech wood by means of ultrasonic waves. Wood Science and Technology, v. 47, n. 2, p. 329-341. 2013.

ROOHNIA, M.; TAJDINI, A.; MANOUCHEHRI, N. Assessing wood in sounding boards considering the ratio of acoustical anisotropy. NDT \& E International, v. 44, n. 1, p. 13-20. 2011.

SEDIK, Y.; HAMDAN, S.; JUSOH, I.; HASAN, M. Acoustic Properties of Selected Tropical Wood Species. Journal of Nondestructive Evaluation, v. 29, n. 1, p. 38-42. 2010.

SHMULSKY, R.; JONES, P. D. Forest Products and Wood Science. Oxford, Wiley: 2011.

SILVA GUZMÁN, J. A.; FUENTES TALAVERA, F. J.; RODRÍGUEZ ANDA R.; TORRES ANDRADE, P. A.; LOMELÍ RAMÍREZ, M. A.; RAMOS QUIRARTE, J.; WAITKUS, C.; RICHTER, H. G. Fichas de propiedades tecnológicas y usos de maderas nativas de México e importadas. México, Comisión Nacional Forestal: 2010.

SMARDZEWSKI, J.; BATKO, W.; KAMISIŃSKI, T.; FLACH, A.; PILCH, A.; DZIURKA, D.; MIRSKI, R.; ROSZYK, E.; MAJEWSKI, A. Experimental study of wood acoustic absorption characteristics. Holzforschung, v. 68, n. 4, p. 467-476. 2014.
SOTOMAYOR CASTELLANOS, J. R. Banco FITECMA de características físico-mecánicas de maderas mexicanas. Morelia, Universidad Michoacana de San Nicolás de Hidalgo: 2015.

SOTOMAYOR CASTELLANOS, J. R.; BOCANEGRA OJEDA, S. Velocidad del ultrasonido en la madera de Picea sp. y de Acer sp. Investigación e Ingeniería de la Madera, v. 5, n. 2, p. 17-26. 2009.

SOTOMAYOR CASTELLANOS, J. R.; GURIDI GÓMEZ, L. I.; GARCÍA MORENO, T. Características acústicas de la madera de 152 especies mexicanas. Velocidad del ultrasonido, módulo dinámico, índice material y factor de calidad. Base de datos. Investigación e Ingeniería de la Madera, v. 6, n. 1, p. 3-32. 2010.

SOTOMAYOR CASTELLANOS, J. R.; RAMÍREZ PÉREZ, M. Densidad y características higroscópicas de maderas mexicanas. Base de datos y criterios de clasificación. Investigación e Ingeniería de la Madera, v. 9, n. 3, p. 3-29. 2013.

SPYCHER, M.; SCHWARZE, F. W. M. R.; STEIGER, R. Assessment of resonance wood quality by comparing its physical and histological properties. Wood Science and Technology, v. 42, n. 4, p. 325-342. 2008.

TAMARIT URIAS, J. C.; LÓPEZ TORRES, J. L. Xilotecnología de los principales árboles tropicales de México. México, Instituto Nacional de Investigaciones Forestales, Agrícolas y Pecuarias: 2007.

TRAORÉ, B.; LOÏC, B.; PERRÉ, P.; STEVANOVIC, T.; DIOUF, P. Acoustic quality of vène wood (Pterocarpus erinaceus Poir.) for xylophone instrument manufacture in Mali. Annals of Forest Science, v. 67, n. 8, p. 815p1-815p7. 2010.

WEGST, U. G. K. Wood for sound. American Journal of Botany, v. 93, n. 10, p. 1439-1448. 2006.

YOSHIKAWA, S. Acoustical classification of woods for string instruments. Journal of the Acoustical Society of America, v. 122, n. 1, p. 568-573. 2007.

YOSHIKAWA, S.; WALTHAM, C. Woods for Wooden Musical Instruments. Proceeding of ISMA 2014. Le Mans. p. 281-286. 2014. 